\title{
Long Noncoding RNA LINC00657 Acting as a miR-590-3p Sponge to Facilitate Low Concentration Oxidized Low-Density Lipoprotein-Induced Angiogenesis
}

\author{
Mei-Hua Bao, Guang-Yi Li, Xiao-Shan Huang, Liang Tang, Li-Ping Dong, and Jian-Ming Li \\ Department of Anatomy, Histology and Embryology, Science Research Center, Institute of Neuroscience (M.-H.B., G.-Y.L., L.T., \\ L.-P.D., J.-M.L.), and Department of Pharmacology (X.-S.H.), Changsha Medical University, Changsha, China
}

Received September 15, 2017; accepted January 29, 2018

\begin{abstract}
Angiogenesis in atherosclerotic plaque promotes plaque growth, causes plaque hemorrhage, and violates plaque stability. LINC00657 is a long noncoding RNA highly conserved and abundantly expressed in vascular endothelial cells. The present study was designed to investigate the effects and mechanisms of LINC00675 on low concentrations of oxidized low-density lipoprotein (oxLDL)-induced angiogenesis. Cell proliferation, transwell, wound healing, and tube formation assays were conducted to detect the effects of low concentrations of oxLDL on angiogenesis; the results discovered that oxLDL promoted cell proliferation, migration, and tube formation. oxLDL also upregulated LINC00657 expression. Inhibition of LINC00657 by siRNA significantly suppressed oxLDL-induced endothelial cell proliferation, migration, and tube formation. Bioinformatic assay indicated six binding sites in the LINC00657 sequence to miR-590-3p. The upregulation of LINC00657 was related to the downregulation of miR-590-3p
\end{abstract}

in oxLDL-treated endothelial cells; while downregulation of LINC00657 resulted in upregulation of miR-590-3p. The antiangiogenesis effects of si-LINC00657 were partly abrogated by miR590-3p inhibitor. Further dual-luciferase assay found miR-590-3p inhibited the expression of hypoxia-inducible factor $1 \alpha(\mathrm{HIF}-1 \alpha)$ by binding to the position of 689-696 in $\mathrm{HIF-1} \alpha 3^{\prime}$-untranslated region directly. MiR-590-3p also inhibited the oxLDL-induced upregulation of HIF- $1 \alpha$, vascular endothelial growth factor (VEGF), matrix metalloproteinase-2 (MMP-2), and matrix metalloproteinase-9 (MMP-9). These results suggested that in oxLDL-treated endothelial cells, LINC00657 acted as a miR-590-3p sponge to attenuate the suppression of miR-590-3p on HIF- $1 \alpha$, and to promote angiogenesis through VEGF, MMP-2, and MMP-9. The present study provided new insight into the roles of LINC00657 and miR-590-3p in preventing oxLDL-induced angiogenesis and may provide a novel strategy for atherosclerosis treatment.

\section{Introduction}

Atherosclerosis is the basis for many cardiovascular and cerebral vascular diseases, such as coronary artery disease, heart infarction, and ischemic stroke (Hellings et al., 2010). The instability and rupture of atherosclerotic plaque are the main reasons for many critical cardiovascular and cerebral vascular events. Recently, studies have demonstrated that the neovascularization in atherosclerotic plaque promoted plaque growth, leukocyte exchange, caused plaque hemorrhage, and violated plaque stability (Camaré et al., 2017; Parma et al., 2017). However, methods to prevent the neovascularization process in atherosclerotic plaque are still limited.

The present study was supported by the Construct Program of the Key Discipline in Hunan Province, National Sciences Foundation of China [Grant 81670427], the Foundation of Hunan Educational Committee [Grants 15C0146, 5B031, and 15A023], Hunan Provincial Natural Science Foundation [Grant 2015JJ6010], and Basic Research Program of Science and Technology Commission Foundation of Hunan Province [Grant 2015JC3059].

https://doi.org/10.1124/mol.117.110650.
In atherosclerotic lesions, local hypoxia happens commonly because of thickening of the vessel wall, which blocks the oxygen exchange, and increased oxygen consumption by high metabolic active cells such as macrophages (Björnheden et al., 1999; Parathath et al., 2011). Hypoxia-inducible factor $1 \alpha$ $(\mathrm{HIF}-1 \alpha)$ is a transcriptional factor activated in the atherosclerotic plaque to adapt to the hypoxic environment. HIF- $1 \alpha$ activation subsequently promotes the production of vascular endothelial growth factor (VEGF), fibroblast growth factor, cytokines, and angiopoietins (Hutter et al., 2013; Perrotta et al., 2015), which results in vascular inflammation and angiogenesis (Deng et al., 2016). Therefore, prevention of HIF$1 \alpha$ might be an effective way to suppress neovascularization in atherosclerosis. Long noncoding RNAs (lncRNAs) are a group of noncoding RNAs with lengths $>200$ nucleotides, but without or with limited ability to encode proteins (International Human Genome Sequencing Consortium, 2004). LINC00657 is a long intergenic noncoding RNA located in the chromosome 20q11.23, with a length of 5,343 base pairs (Liu et al., 2016). Studies have shown that LINC00657 is a highly conserved and abundantly

ABBREVIATIONS: DAPI, 4'6-diamidino-2-phenylindole; ELISA, enzyme-linked immunosorbent assay; HIF-1 $\alpha$, hypoxia-inducible factor $1 \alpha$; HUVEC, human umbilical vascular endothelial cell; IncRNA, long noncoding RNA; MMP-2, matrix metalloproteinase-2; MMP-9, matrix metalloproteinase-9; MTS, [3-(4,5-diethylthiazol-2-yl)-5-(3-carboxymethoxyphenyl)-2-(4-sulfophenyl)-2H-etrazolium,inner salt]; oxLDL, oxidized low-density lipoprotein; PCR, polymerase chain reaction; si-NC, negative control siRNA; UTR, untranslated region; VEGF, vascular endothelial growth factor. 
expressed lncRNA in endothelial cells (Michalik et al., 2014). The expression of LINC00657 was upregulated after hypoxia (Michalik et al., 2014), which inspires us to presume that LINC0657 might participate in neovascularization in atherosclerosis.

MicroRNAs are another important group of noncoding RNAs with lengths of 18-24 nucleotides. Our previous studies have demonstrated the important roles of microRNAs in atherosclerosis (Bao et al., 2016a,b). MiR-590-3p is a microRNA located on the chromosome 7 q11.23. MiR-590-3p was demonstrated to be involved in cancers, lipid metabolism, inflammation, and cardiac regeneration (Eulalio et al., 2012; Ge and Gong, 2017). However, whether miR-590-3p participates in angiogenesis remains unclear. The previous study indicated that low concentrations of oxidized low-density lipoprotein (oxLDL) promoted angiogenesis (Yu et al., 2011). Our pilot experiments found oxLDL treatment inhibited the expression of miR-590-3p and increased the expression of LINC00657. Bioinformatics has shown that LINC00657 contains six binding sites for miR-590-3p, which intrigues us to assume that LINC00657 might act as a miR-590-3p sponge. The present study was performed to investigate the effects and mechanisms of LINC00657 and miR-590-3p on angiogenesis in human umbilical vascular endothelial cells (HUVECs) induced by low concentrations oxLDL.

\section{Materials and Methods}

Materials. The HUVEC cell line was provided by the American Type Culture Collection (Manassas, VA). Dulbecco's modified Eagle's medium was obtained from Sigma (St. Louis, MO). The oxLDL was supplied by ThermoFisher Scientific (Grand Island, NY). SYBR Green Premix DimerEraser kits and PrimeScript RT Reagent Kit with gDNA Eraser (Perfect Real Time) kits were purchased from Takara (Dalian, China). The primers were provided by Shanghai Sangon Biologic Engineering Co. Ltd. (Shanghai, China). Mouse monoclonal antibody to HIF-1 $\alpha$ was purchased from Abcam (Cambridge, MA) and rabbit monoclonal antibody to VEGFR2 and to $\beta$-actin were purchased from Cell Signaling Technology (Danvers, MA). DAPI (4'6-diamidino-2phenylindole) was obtained from Beyotime Institute of Biotechnology (Shanghai, China). Corning Matrigel Basement Membrane Matrix was provided by Corning (Corning, NY). The si-LINC00657, negative control siRNA (si-NC), and miR-590-3p mimics or inhibitor were obtained from RiboBio Co., Ltd. (Guangzhou, China). Secrete-Pair Dual Luminescence Assay Kit was provided by GeneCopoeia (Rockville, MD). pMIRREPORT Luciferase Vector was provided by ThermoFisher Waltham, (Waltham, MA). The VEGF, matrix metalloproteinase-2 (MMP-2) and matrix metalloproteinase-9 (MMP-9) enzyme-linked immunosorbent assay (ELISA) kit were purchased from Bosterbio Corp. (Wuhan, China).

Cell Culture and Transfection. HUVECs were cultured in Dulbecco's modified Eagle's medium (low glucose) with $10 \%$ fetal bovine serum. The cultivation environment was humidified atmosphere with $5 \% \mathrm{CO}_{2}$ at $37^{\circ} \mathrm{C}$. Cells between passages 2 and 15 were used in this study.

For the transfection, lipofectamine 2000 was used to transfect miR-590-3p mimics, miR-590-3p inhibitor, si-LINC00657, and plasmid DNA. Briefly, the lipofectamine 2000, plasmid DNA, miR-590-3p mimics, and miR-590-3p inhibitor were diluted in Opti-MEM I Medium (ThermoFisher) and incubated for 5 minutes. After incubation, the diluted lipofectamine 2000 was blended with diluted plasmid DNA, miR-590-3p mimics, and miR-590-3p inhibitor, and then incubated for another 20 minutes at room temperature. The transfection mixtures were then added to cells and incubated for 6 hours. After that, the medium was replaced by fresh normal growth medium. The siRNAs in the present study are si-LINC00657: $5^{\prime}$-TAG CCC TTC TAG ATG GAA A-3'; and si-NC: 5'-GCG CGA TAG CGC GAA TAT A-3', as reported previously (Lee et al., 2016).
Cell Proliferation Assay. For the cell proliferation assay, HUVECs were cultivated in a 96-well plate at a density of $5 \times 10^{3}$ cells per well. After treatment by different concentrations of oxLDL $(0,5,10$, and $50 \mu \mathrm{g} / \mathrm{ml})$ for different time periods $(0,12,24$, and 48 hours), the reagent MTS (3-(4,5-diethylthiazol-2-yl)-5-(3-carboxymethoxyphenyl)2-(4-sulfophenyl)-2H-etrazolium,inner salt) (ThermoFisher) was added to each well according to the manufacturer's instructions. Cell proliferation was assessed by measuring absorbance at $490 \mathrm{~nm}$ using a plate reader.

Wound Healing Assay. The effects of LINC00657 on HUVEC migration were assessed by wound healing assays. The HUVECs were cultured in a 12 -well plate at a density of $5 \times 10^{5}$ cells/well. Cells were transfected with si-LINC00657 $(1.2 \mu \mathrm{g})$ alone or with miR-590-3p inhibitor (100 nM) for 24 hours, and then a yellow pipette tip was used to scrape a wound gap. The cells were carefully washed with phosphate-buffered saline and treated with $10 \mu \mathrm{g} / \mathrm{ml}$ oxLDL for another 48 hours. Three randomly selected regions were recorded using a microscope. The distance between cell boundaries was measured and the percentage of the wound gap that had been healed was calculated.

Transwell Assay. To further assess the effects of LINC00657 on endothelial cell migration, transwell assay was performed. HUVECs were transfected first with si-LINC00657 $(1.2 \mu \mathrm{g})$ alone or with miR-590-3p inhibitor (100 nM) for 24 hours as described in Cell Culture and Transfection, and then collected and added to the upper chamber of the transwell in serum-free medium $\left(4 \times 10^{4}\right.$ endothelial cells per chamber). The lower chamber was filled with Dulbecco's modified Eagle's medium with $20 \%$ fetal bovine serum. Then, oxLDL with an end concentration of $10 \mu \mathrm{g} / \mathrm{ml}$ was added to the upper chamber, and incubation for another 48 hours was performed. At the end of the incubation, cells were stained with DAPI (4',6-diamidino-2phenylindole), and then cells on the lower surface of the filter were photographed and counted under a fluorescence microscope.

Tube Formation Assay. The capability of HUVECs to form capillary tube-like structures was assessed by the Matrigel-based tube formation assay as previously described (Gonzalez-King et al., 2017). First, HUVECs were transfected with si-LINC00657 and miR$590-3 \mathrm{p}$ inhibitor ( $100 \mathrm{nM})$ for 24 hours as described in Cell Culture and Transfection. Then, $50 \mu \mathrm{l}$ of Matrigel was added to a 96 -well plate and solidified at $37^{\circ} \mathrm{C}$ for 1 hour. After that, the pretransfected HUVECs were harvested and cultured on the Matrigel-coated plate for another 24 hours, with or without oxLDL treatment. The tube formation effects were observed and photographed through the microscope. The tube-like structure length was calculated.

Real-Time Polymerase Chain Reaction (PCR) for Detection of LINC00657, miR-590-3p, HIF1 $\alpha$, VEGF, MMP-2, and MMP-9. As described previously (Bao et al., 2016a,b), total RNA was extracted using TRIzol reagent (ThermoFisher). Then, the total RNA was reversely transcripted to the complementary DNA using the RT Reagent Kit provided by Takara according to the manufacturer's instructions. Real-time PCR amplification reactions were conducted using the SYBR Premix DimerEraser (Perfect Real Time) assay kits and the TL988-IV System (Tianlong, Xi'an, China). The real-time quantitative PCR program was an initial incubation at $95^{\circ} \mathrm{C}$ for 30 seconds, followed by 40 cycles of $95^{\circ} \mathrm{C}$ for 5 seconds and $60^{\circ} \mathrm{C}$ for 30 seconds. The PCR primers are described as follows: LINC00657: forward: 5' - TGA TAG GAT ACA TCT TGG ACA TGG A -3', reverse: $5^{\prime}$ - AAC CTA ATG AAC AAG TCC TGA CAT ACA-3'; HIF1 $\alpha$ : forward: $5^{\prime}$-CGT CGC TTC GGC CAG TGT GT $-3^{\prime}$, reverse: $5^{\prime}$-TCC AGA GGT GGG GGT GCG AG-3'; MMP-2: forward: 5' -GTT TCC ATT CCG CTT CCA GG-3', reverse: 5'-TGC CCT TGA TGT CAT CCT GG-3'; MMP-9: forward: 5'-GAC CTC AAG TGG CAC CAC CA-3', reverse: $5^{\prime}$-GTG GTA CTG CAC CAG GGC AA-3'; and $\beta$-actin: forward: $5^{\prime}$-TGA CTG ACT ACC TCA TGA AGA T-3', reverse: $5^{\prime}$-CAT GAT GGA GTT GAA GGT AGT T-3' . All samples were run in triplicate, and the results were analyzed using the $2^{(-\Delta \Delta \mathrm{Ct})}$ method. For miR590-3p, U6 was used as the internal reference, while for other genes, $\beta$-actin was used. 
A

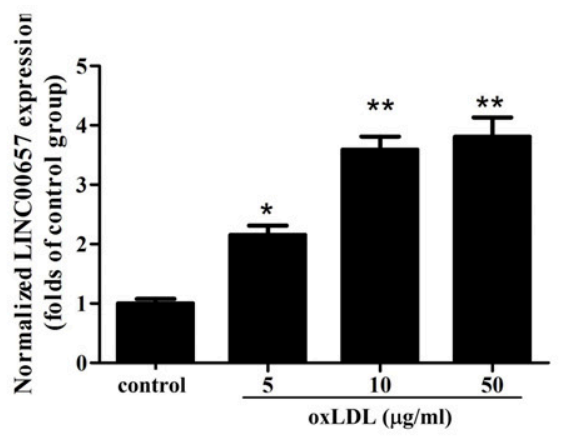

C

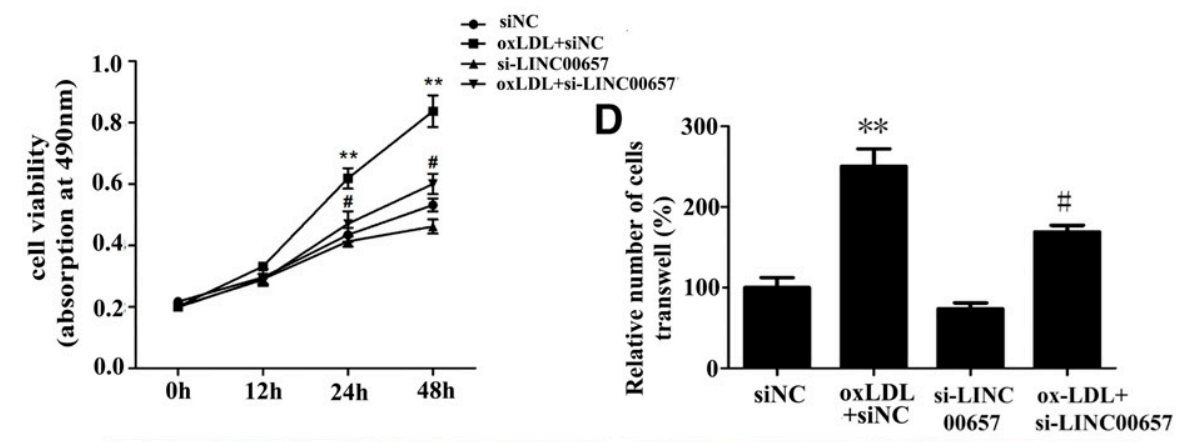

E

Transwel
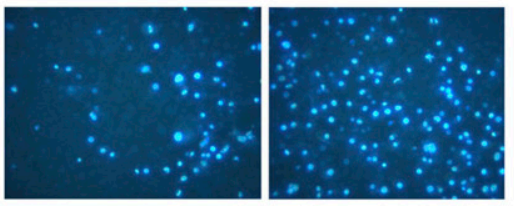

F

Tube formation

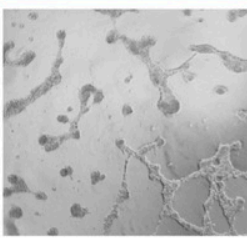

G

H
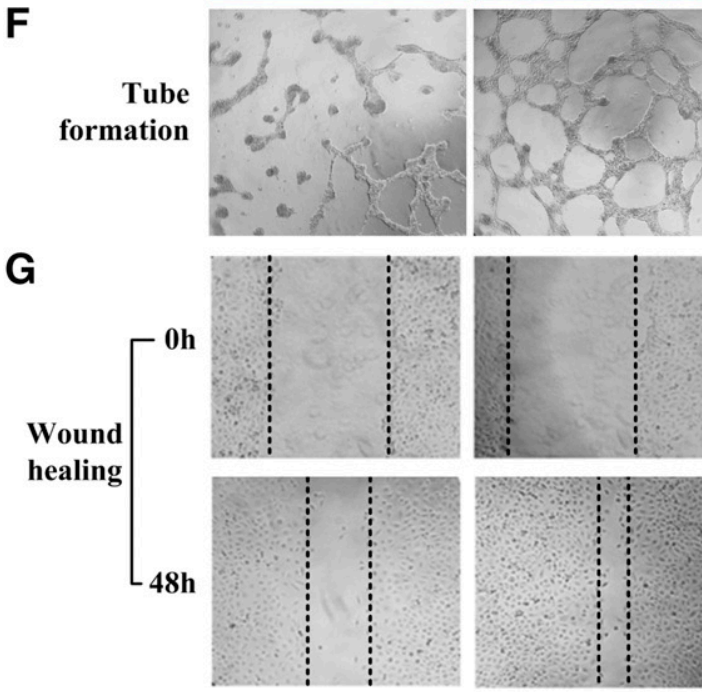

siNC

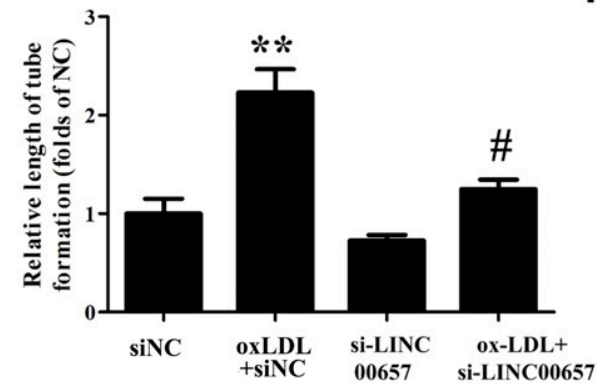

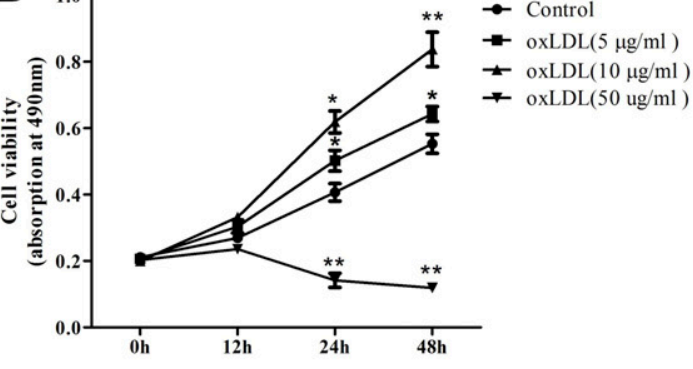

B $\left.\quad{ }^{1.0}\right]$
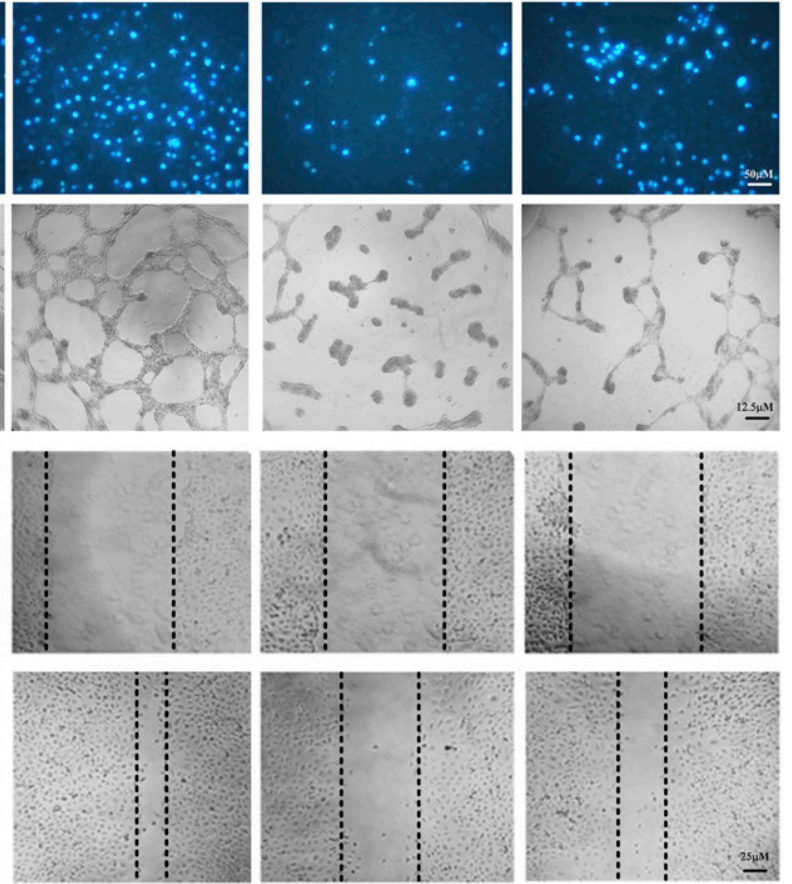

oxLDL+siNC

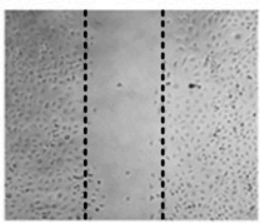

si-LINC00657
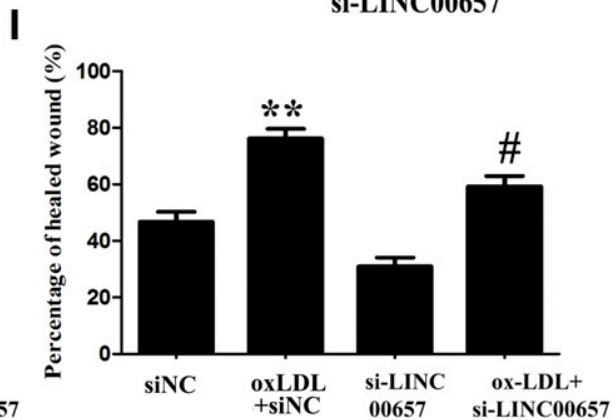

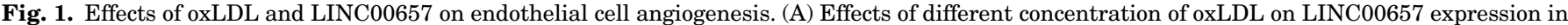

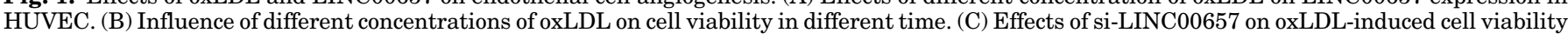


Western Blot Assay for HIF-1 $\alpha$. HUVECs were cultured and transfected as described in Cell Culture and Transfection. After transfection, cells were treated with oxLDL $(10 \mu \mathrm{g} / \mathrm{ml})$ for another 24 hours. After the treatment, the cells were collected and washed twice with phosphate-buffered saline. The total proteins were extracted by adding $50 \mu \mathrm{l}$ radioimmunoprecipitation assay lysis (with $1 \%$ phenylmethylsulfonyl fluoride and $10 \mathrm{mM} \mathrm{NaF}$ ) into the cells for 30 minutes.

For the western blot assay, the total proteins were separated by SDS-PAGE and transferred to polyvinylidene fluoride membranes. After that, the membranes were blocked with $4 \%$ nonfat milk for 1 hour and then incubated with diluted HIF- $1 \alpha$ primary antibody overnight at $4^{\circ} \mathrm{C}$. The membranes were afterward washed and incubated with 1:10,000 dilution of the second antibody for 1 hour and were detected with the enhanced chemiluminescence system. Relative intensities were analyzed by Quantity One ${ }^{R}$ software (Bio-Rad, Hercules, CA).

ELISA Detection of VEGF, MMP-2, and MMP-9 Levels. HUVECs were cultured and transfected as described in Cell Culture and Transfection. After transfection, cells were treated with oxLDL (10 $\mu \mathrm{g} / \mathrm{ml})$ for another 24 hours. After treatment, the culture supernatants were collected and the level of VEGF, MMP-2, and MMP-9 were measured by the ELISA assay kits, following the manufacturer's instructions.

HIF-1 $\alpha$ 3'-Untranslated Region [(UTR) Wild-Type and Mutant] Reporter Plasmid Construction, Transfection, and Dual Luciferase Reporter Analysis. The PCR was performed using primers specific for the HIF-1 $\alpha 3^{\prime}$-UTR (forward: $5{ }^{\prime}$-GCCG CTC GAG GCT TTT TCT TAA TTT CAT TCC TTT TTTT GG-3'; reverse: 5'-GAA TGC GGC CGC GCC TGG TCC ACA GAA GAT GTT TAT TTG A-3'). The forward primers included an Xhol cutting site and the reverse primer contained a Notl cutting site. HUVEC genomic DNA was used as the template. The PCR products were digested with Xhol and Notl and cloned to pMIR-REPORT luciferase vector (Ambion [ThermoFisher]). The mutation at the potential binding site of miR590-3p was performed using the QuikChange Site-Directed Mutagenesis Kit (Stratagene, La Jolla, CA). The predicted miR-590-3p binding sites (underlined) were changed as follows: wild type, $5^{\prime}$-UGGCAUUUAUUUGGAUAAAAUUC-3'; mutant type: 5'-UGGCAUUUAUUU GGAGACA CUCC-3'.

For the transfection, HUVECs were cultured in a $\overline{24 \text {-well plate }}$ at a density of $5 \times 10^{4}$ cells/well for 24 hours, and then $400 \mathrm{ng}$ of wild-type, mutant, or blank plasmid DNA was cotransfected with $100 \mathrm{nM}$ miR-590-3p or negative control mimics. Dual luciferase reporter analysis was performed at 48 hours post-transfection using the Luciferase Assay kit according to the manufacturer's instructions (Promega, Madison, WI).

Statistical Analysis. All statistics from three independent experiments are presented in the form of mean \pm S.D. values. The significance of the differences was analyzed by analysis of variance, followed by the Newman-Student-Keuls test. A value of $P<0.05$ is considered statistically significant.

\section{Results}

Effects of oxLDL on Cell Viability and LINC00657 Expression. LINC00657 was thought to be highly expressed in endothelial cells (Michalik et al., 2014). Our present study also found a high expression of LINC00657 in HUVECs (Fig. 1A). After 24-hour treatment with different concentrations of oxLDL, the LINC00657 expression increased significantly
$(P<0.05$ vs. control). Relatively low concentrations of oxLDL (5 and $10 \mu \mathrm{g} / \mathrm{ml}$ ) treatment promoted the proliferation of HUVECs during the culture period, while a high concentration of oxLDL $(50 \mu \mathrm{g} / \mathrm{ml})$ inhibited the proliferation of HUVECs (Fig. 1B).

Effects of LINC00657 on oxLDL-Induced Angiogenesis. In the present study, cell viability, wound healing, transwell, and tube formation assays were conducted to measure the function of LINC00657 on low concentrations oxLDL-induced angiogenesis. As shown in Fig. 1C, 24- and 48-hour treatment with oxLDL $(10 \mu \mathrm{g} / \mathrm{ml})$ stimulated the growth of HUVECs significantly, and LINC00657 inhibition by siRNA suppressed the cell growth induced by oxLDL. Transwell assay found that 48-hour treatment with oxLDL promoted the migration of HUVECs from the upper to the lower chamber (about 2.5-fold of control), and si-LINC00657 inhibited oxLDL-induced cell migration (Fig. 1, D and E). We also found oxLDL facilitated the tube formation and wound healing of HUVECs, and si-LINC00657 suppressed the alteration (Fig. 1, F-I).

si-LINC00657 Promoted the Expression of miR-5903p. To explore the mechanisms of LINC00657, we analyzed its sequence and found it contained six binding sites with miR590-3p (Fig. 2A). Therefore, we assessed whether LINC00657 acts as a competitive endogenous RNA to miR-590-3p and inhibits the expression of miR-590-3p. As shown in Fig. 2B, si-LINC00657 significantly downregulated the level of LINC00657 (32.9\% of control group). The inhibition of LINC00657 resulted in a dramatic induction of miR-590-3p (4.5-fold of control) (Fig. 2C). Treatment with oxLDL $(10 \mu \mathrm{g} / \mathrm{ml})$ for 24 hours promoted the expression of LINC00657, while it downregulated the expression of miR-590-3p (Fig. 2D).

Inhibition of miR-590-3p Partly Abrogated the Effects of LINC00657 on oxLDL-Induced Angiogenesis. To verify whether the antiangiogenesis effects of LINC00657 are mediated by miR-590-3p, we detected the effects of miR590-3p inhibitor on antiangiogenesis of si-LINC00657. The inhibition of miR-590-3p by inhibitor abrogated the suppression of angiogenesis by si-LINC00657 in oxLDL-treated HUVECs (Fig. 3A). We also found si-LINC0657 suppressed endothelial cell transwell migration, tube formation, and wound healing. All of these effects were suppressed to a certain degree by miR-590-3p inhibition (Fig. 3, B-G). These results indicate that si-LINC00675 might play a role in angiogenesis through miR-590-3p.

Target Identification for miR-590-3p. To figure out the mechanism for the effects of miR-590-3p, we predicted the targets for miR-590-3p by bioinformatics. As shown in Fig. 4B, miR-590-3p is predicted to bind to position $689-696$ of HIF-1 $\alpha$ $3^{\prime}$-UTR. To verify this prediction, a dual luciferase reporter assay was performed. The results showed that in HUVECs, miR-590-3p mimics significantly inhibited the luciferase activity, while miR-590-3p inhibitor promoted it (Fig. 4A). The mutation of the binding position of HIF- $1 \alpha$ eliminated the effects of miR-590-3p. To further verify the regulation of miR-590-3p to HIF- $1 \alpha$, we tested the HIF- $1 \alpha$ levels after

change; cell viability at different times. HUVECs were transfected with si-LINC00657 or siNC for 24 hours and then treated with oxLDL (10 $\mu \mathrm{g} / \mathrm{ml}$ ) for the indicated time. Cell viability was assessed by the MTS method. (D and E) Transwell assay for cell migration. HUVECs were transfected with si-LINC00657 (100 nM) or siNC for 24 hours and then treated with oxLDL $(10 \mu \mathrm{g} / \mathrm{ml})$ for another 48 hours. The cells in the lower chamber were stained with DAPI (4',6-diamidino-2-phenylindole) and calculated. (F) Tube formation assay. (G) Wound healing assay. A linear wound was made, and oxLDL and/or si-LINC00657 were added, and the distance between the cell boundaries was measured. $(\mathrm{H})$ Relative length of endothelial cell tube formation. Tube length was quantified in three random microscope fields, and normalized relative to the negative control group; (I) Percentage of the healed wound. Data are mean \pm S.D. values from three independent experiments; ${ }^{*} P<0.05 ;{ }^{* *} P<0.01$, compared with NC; ${ }^{*} P<0.05$ compared with the oxLDL group. 
A

\begin{tabular}{|c|c|}
\hline miR-590-3p & $\begin{array}{c}\text { 3'- TGATCGAATATGT - ATTTTAAT-5' } \\
|||||||||||| \mid\end{array}$ \\
\hline $\begin{array}{r}\text { LINC00657 } \\
1202 \text { bp-1223bp }\end{array}$ & 5' - ...AGTGCCTGATATGTTAAAATTA...-3' \\
\hline miR-590-3p & $\begin{array}{l}\text { 3'- TGATCGAATATGTATTTTAAT-5' } \\
||||||||||||\end{array}$ \\
\hline 1829 bp-1849 & АATATC \\
\hline
\end{tabular}

$$
\begin{aligned}
& \text { miR-590-3p 3'-TGATCGAATAT GTATTTTAAT-5' } \\
& \text { LINC00657 | | | ||||||| }
\end{aligned}
$$

ATTTTAAT-5

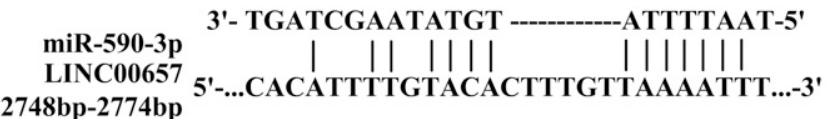

$$
\begin{array}{rc}
\text { miR-590-3p } & \text { 3'-TGATCGAATATGTATTTTAAT-5' } \\
\text { LINC00657 } & 5 \text { '-...AGTCTCAGTCCCATAAAATTG...-3' }
\end{array}
$$
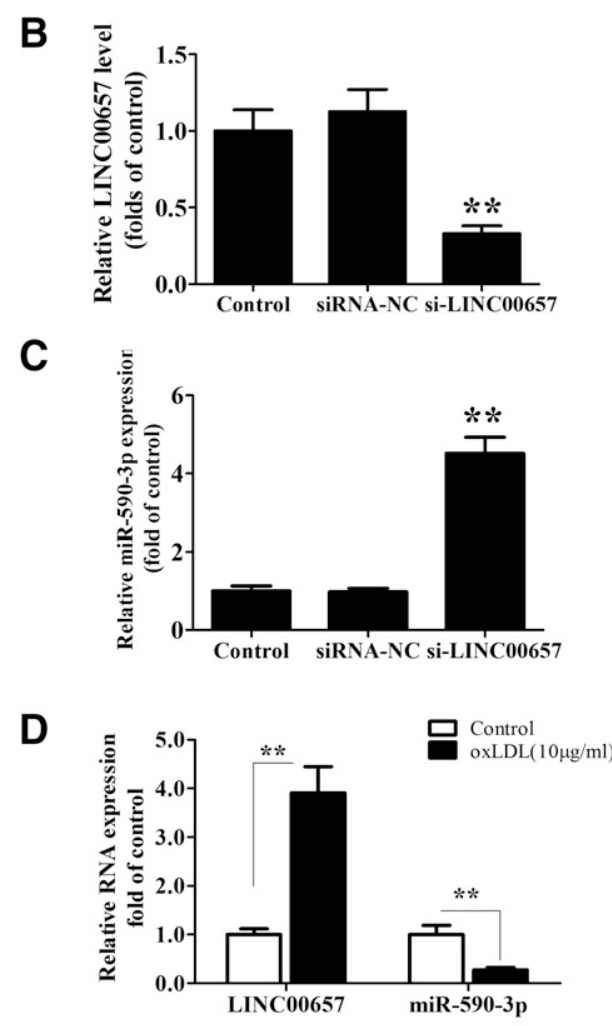

Fig. 2. Regulation of LINC00657 on miR-590-3p expression. (A) Alignment of LINC00657 and miR-590-3p; (B) effects of si-LINC00657 on LINC00657 expression. HUVECs were cultured in 24-well plates and transfected with $500 \mathrm{ng}$ of si-LINC00657. The expression of LINC00657 was measured by quantitative PCR. (C) Effects of si-LINC00657 on miR-590-3p expression. (D) Expression of LINC00657 and miR-590-3p after oxLDL treatment for 24 hours. Data are mean \pm S.D. values from three independent experiments; $* * P<0.01$, compared with control.

miR-590-3p overexpression and inhibition. As shown in Fig. 4, C-E, the overexpression of miR-590-3p in HUVECs inhibited the expression of HIF-1 $\alpha$ both in mRNA and protein level.

Effects of miR-590-3p on HIF1 $\alpha$, VEGF, MMP-2, and MMP-9 Expression. HIF- $1 \alpha$ is widely considered to be a transcriptional factor that regulates many angiogenesisrelated genes, such as VEGF and MMPs. In the present study, quantitative PCR, western blot, and ELISA were used to investigate the effects of miR-590-3p on HIF-1 $\alpha$, VEGF, MMP-2, and MMP-9 mRNA and protein expression with and without oxLDL. As shown in Fig. 4, F-I, miR-590-3p significantly suppressed both the mRNA and protein levels of HIF-1 $\alpha$, VEGF, MMP-2, and MMP-9 in HUVECs. When the endothelial cells were treated with low concentrations of oxLDL, the expression of HIF-1 $\alpha$, VEGF, MMP-2, and MMP9 increased; miR-590-3p overexpression suppressed the oxLDL-induced increase of these factors.

\section{Discussion}

Our present study found that low concentrations of oxLDL promoted the proliferation, migration, and tube formation of HUVECs, as well as the expression of LINC00657. Inhibition of LINC00675 by siRNA suppressed oxLDL-induced angiogenesis and expression of miR-590-3p. Moreover, the effects of si-LINC00657 were partly abrogated by the miR590-3p inhibitor. Further studies found that miR-590-3p inhibited the expression of HIF-1 $\alpha$ by directly targeting the position of $689-696$ in HIF- $1 \alpha 3^{\prime}$-UTR. The inhibition of HIF$1 \alpha$ subsequently decreased the production of VEGF, MMP-2, and MMP-9, which may contribute to the angiogenesis caused by low concentrations of oxLDL during atherosclerosis.

oxLDL has long been considered as a major risk factor for endothelial cell dysfunction and death in atherosclerosis. However, studies also found low concentrations of oxLDL promoted angiogenesis (Dandapat et al., 2007; Yu et al., 2011; Khaidakov et al., 2012). VEGF expression, redox-sensitive pathways, and nitric oxide synthesis were thought to be involved in the proangiogenesis effects of oxLDL (Dandapat et al., 2007; Yu et al., 2011; Khaidakov et al., 2012). Our present study verified that low concentrations of oxLDL (5 and $10 \mu \mathrm{g} / \mathrm{ml}$ ) promoted the growth of HUVECs, and $10 \mu \mathrm{g} / \mathrm{ml}$ of oxLDL induced the transwell migration, tube formation, and wound healing characteristics of endothelial cells. Furthermore, we found that treatment with low concentrations of oxLDL $(10 \mu \mathrm{g} / \mathrm{ml})$ increased the production of proangiogenesis factors, such as VEGF, MMP-2, and MMP-9. VEGF is a strong stimulator for both physiologic and pathologic angiogenesis (Ribatti et al., 2001). MMP-2 and MMP-9 are the two most important members of MMP family for extracellular matrix degradation, which facilitate endothelial cell migration and angiogenesis (Liu et al., 2014). In our study, we found that low concentrations of oxLDL increased the expression of VEGF, MMP-2, and MMP-9, consistent with previous reports (Dandapat et al., 2007; Tsai et al., 2016). These results might partly explain the proangiogenesis effects of low concentration oxLDL on endothelial cells. 
A

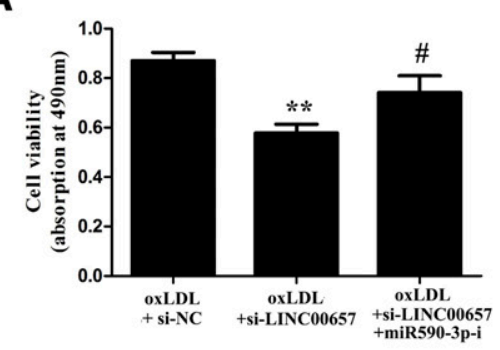

B

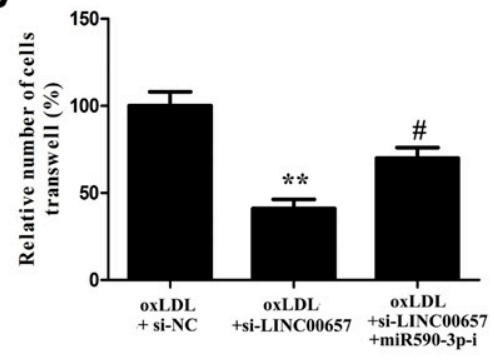

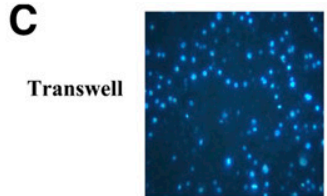

D

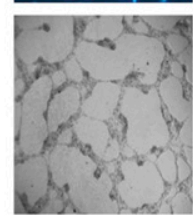

\section{E}

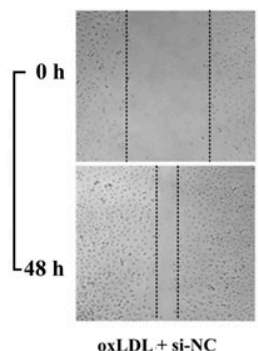

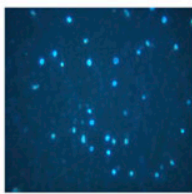
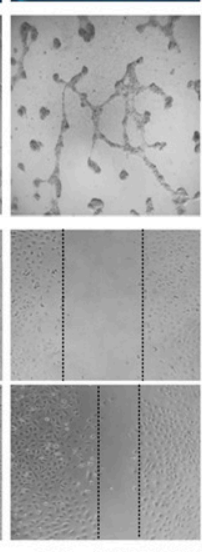

oxLDL+si-LINC00657
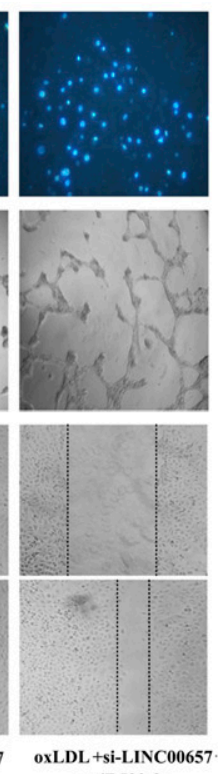

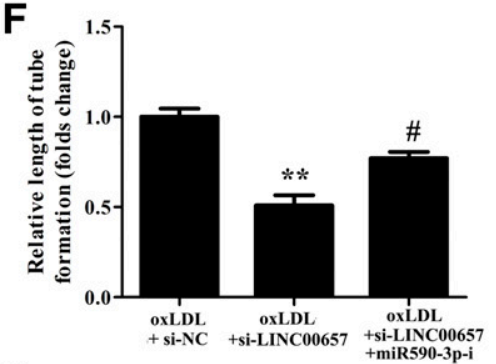

G

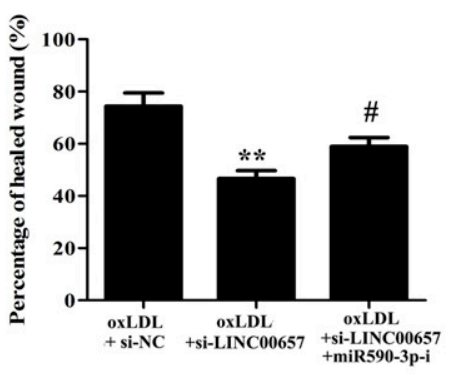

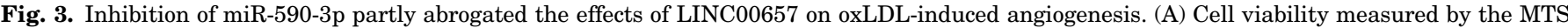

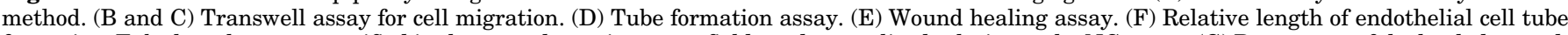

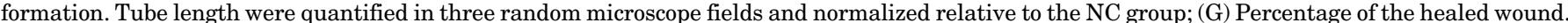

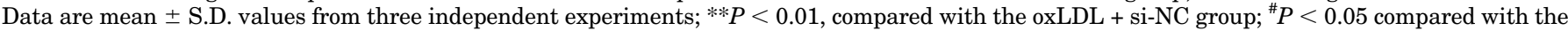
oxLDL + si-LINC00657 group.

To explore the mechanisms of oxLDL-induced angiogenesis, we focused on one lncRNA, LINC00657. LINC00657, also called noncoding RNA activated by DNA damage, is a $5.3 \mathrm{~kb}$ intergenic long noncoding RNA broadly and abundantly expressed in human tissues, including endothelial cells (Michalik et al., 2014; Lee et al., 2016; Liu et al., 2016). The high expression of LINC00657 is related to breast cancer cell growth and proliferation (Liu et al., 2016). In endothelial cells, LINC00657 is highly expressed in the cytoplasm (Michalik et al., 2014). Hypoxia $\left(0.2 \% \mathrm{O}_{2}\right)$ induced the expression of LINC00657 to 2.5-fold compared with normoxia (Michalik et al., 2014). It should be noted that oxLDL is an oxidatively modified product of low-density lipoprotein, and oxLDL results from oxidative burst during infection or other oxidative pathologic processes. On the other hand, oxLDL also promotes oxidative stress, which is involved in the pathogenesis of atherosclerosis (Ishigaki et al., 2009; Peluso et al., 2012). Since hypoxia and high levels of oxLDL exist in the atherosclerotic lesion, we therefore presumed that LINC00657 might participate in the pathogenesis of atherosclerosis, and that oxLDLinduced oxidative stress may cause altered expression of LINC00657. Our present study found a significant increase in LINC00657 expression after oxLDL treatment. The inhibition of LINC00657 by siRNA suppressed oxLDLinduced cell proliferation, migration, tube formation, and wound healing ability. These results demonstrated that LINC00657 is involved in the proangiogenesis effects of oxLDL. However, the details of the mechanism of how hypoxia or oxLDL promote LINC00657 expression are still unclear.

The lncRNAs play their roles through many mechanisms including transcriptional and post-transcriptional regulation, sources of small RNAs, miRNA sponges, mRNA binders, protein binders, etc.(Kornienko et al., 2013). The bioinformatic analysis found six binding sites on LINC00657 with miR-590-3p.
LINC00657 is reported to exist in the cytoplasm (Michalik et al., 2014). We found that inhibition of LINC00657 by siRNA promoted the expression of miR-590-3p, while the increase of LINC00657 in oxLDL-treated endothelial cells was related to the decrease of miR-590-3p. Further research found miR-590-3p inhibitor abrogated the effects of si-LINC00657 on oxLDL-induced angiogenesis. These results demonstrated that LINC00657 might interact with miR-590-3p and interfere with the process of angiogenesis.

miR-590-3p is a microRNA located at the position of chromosome $7 \mathrm{q} 11.23$. The functions of miR590 have been discovered in recent years. Both miR590-5p and miR-590-3p were found to participate in the carcinogenesis of different cancers, such as prostate cancer, liver cancer, lung cancer, etc. (Wang et al., 2016; Chen et al., 2017; Ge and Gong, 2017). A most recent study found miR590-5p inhibited colorectal cancer angiogenesis through the NF90/VEGFA signal (Zhou et al., 2016). However, the effects of miR-590-3p on angiogenesis are still unclear. Our study found a significant decrease of miR-590-3p in HUVECs after oxLDL treatment. Bioinformatics predicts miR-590-3p may bind to the position of 689696 in HIF-1 $\alpha$ 3'-UTR. Further dual luciferase reporter analysis and western blot analysis verified the interaction between miR-590-3p and HIF- $1 \alpha$.

HIF- $1 \alpha$ is a transcriptional factor reported to be activated by oxLDL. The activation of HIF-1 $\alpha$ promotes the expression of many growth factors and cytokines, regulates cell proliferation and survival, and is involved in biologic processes such as angiogenesis (Lee et al., 2004; Sun et al., 2010). Therefore, the regulation of miR-590-3p on HIF- $1 \alpha$ might be responsible for the effects of miR-590-3p on angiogenesis. Our present results demonstrated an increase in HIF-1 $\alpha$ expression after oxLDL treatment, which subsequently increased the production of VEGF, MMP-2, and MMP-9. Also, mir-590-3p upregulation inhibited oxLDL-induced HIF-1 $\alpha$, VEGF, MMP-2 and MMP-9 


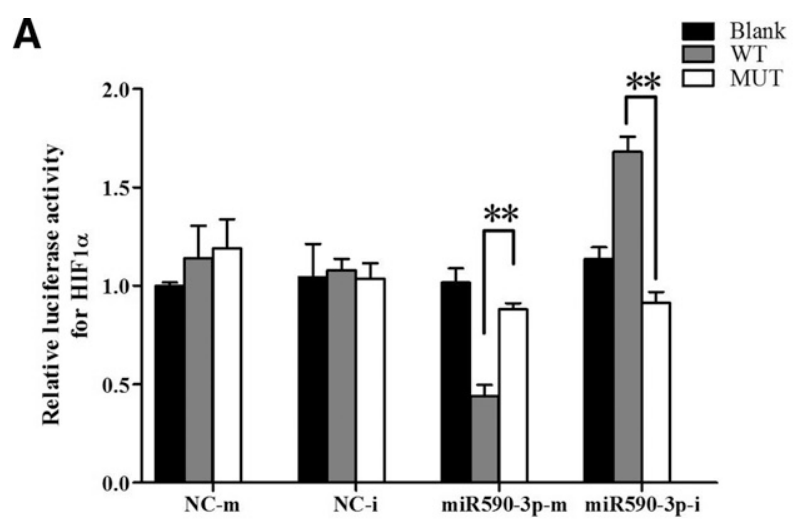

B

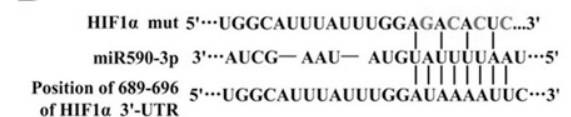

D

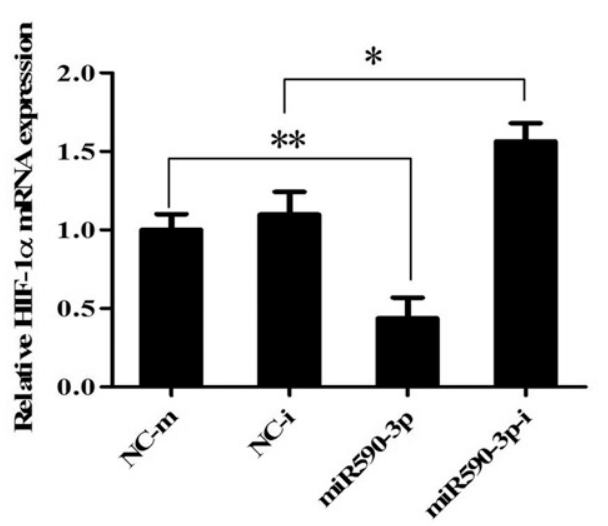

C
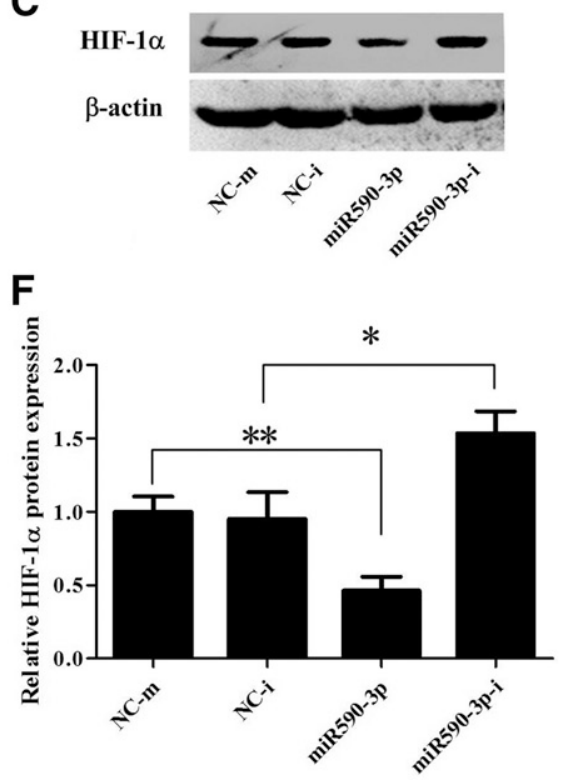

F ${ }^{4.0}{ }^{\mathrm{N} C-\mathrm{m}} \mathrm{miR590-3 \textrm {p }}$

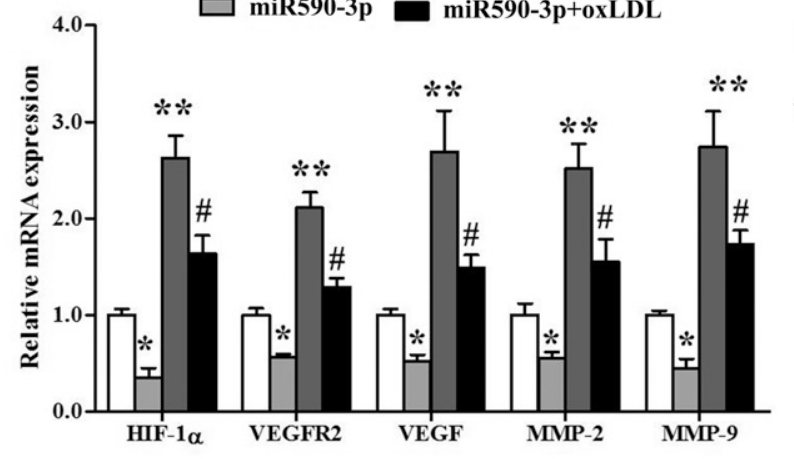

G

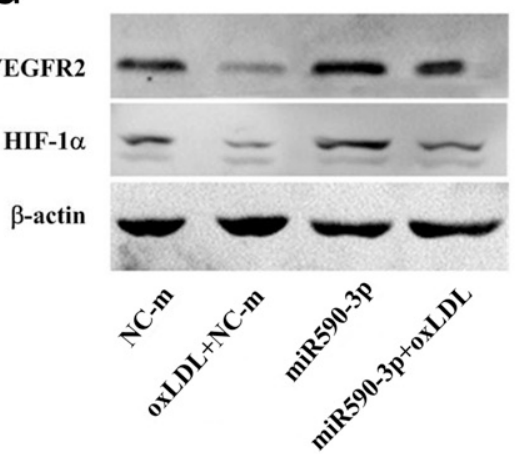

H

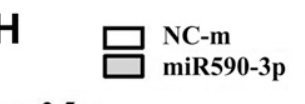

oxLDL+NC-m miR590-3p+oxLDL

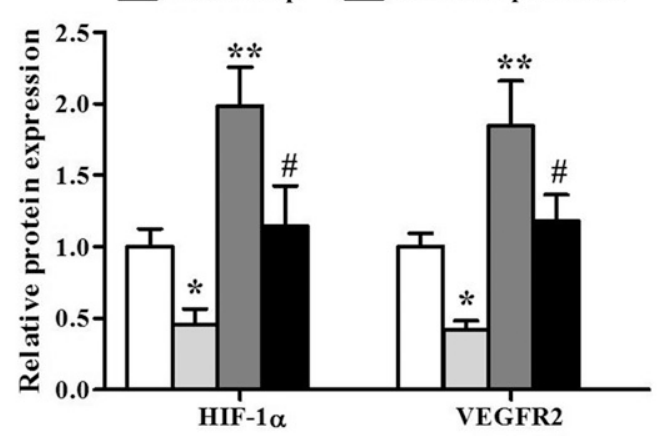

I

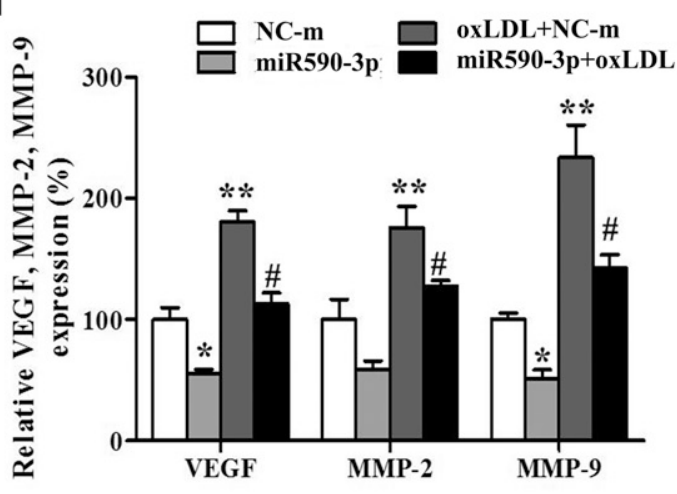

Fig. 4. The regulation of miR-590-3p on HIF-1 $\alpha$, VEGF, MMP-2, and MMP-9 expression. (A) Relative luciferase activities measured by dual luciferase reporter analysis. (B) Predicted miR-590-3p binding site in HIF-1 $\alpha$. (C) Effects of miR-590-3p on HIF-1 $\alpha$ protein expression. (D) mRNA expression of HIF-1 $\alpha$. (E) Normalized level of HIF-1 $\alpha$ protein. (F) Effects of miR-590-3p on oxLDL-induced HIF-1 $\alpha$, VEGF, MMP-2, and MMP-9 mRNA expression; (G) effects of miR-5903 p on oxLDL-induced HIF-1 $\alpha$ protein expression; (H) normalized level of HIF- $1 \alpha$ and VEGFR2 protein; (I) ELISA analysis of VEGF, MMP-2, and MMP-9 levels in HUVEC culture supernatant. Data are mean \pm S.D. values from three independent experiments; ${ }^{*} P<0.05$; ${ }^{*} P<0.01$ vs. NC; ${ }^{*} P<0.05$ vs. oxLDL. 


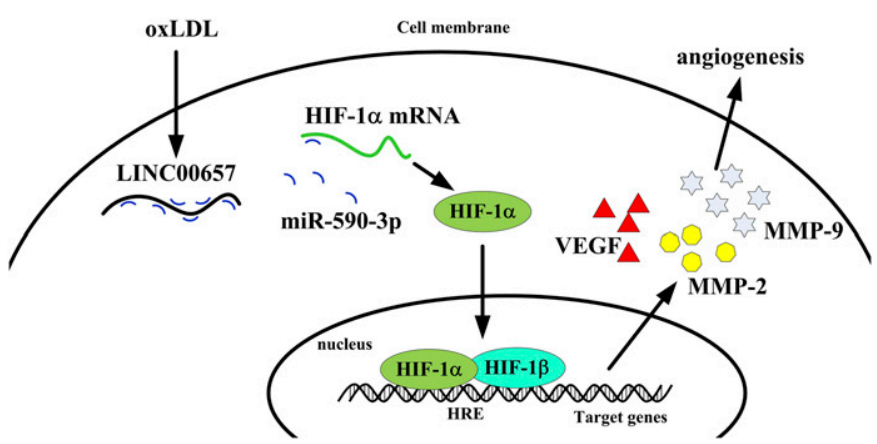

Fig. 5. Function of LINC00657 in oxLDL-induced angiogenesis. In endothelial cells, low concentrations of oxLDL promotes the expression of LINC00657; the LINC00657 acts as a miRNA sponge and inhibits the level of miR-590-3p; miR-590-3p loses its suppression on HIF-1 $\alpha$ and leads to the upregulation of HIF- $1 \alpha$; HIF- $1 \alpha$ then promotes the expression of VEGF, MMP-2, and MMP-9, and eventually induces angiogenesis.

expression, indicating the functionalities of miR-590-3p might be partly obtained through the HIF- $1 \alpha$ signal.

In conclusion, our results suggest that in endothelial cells, low concentrations of oxLDL promote the expression of LINC00657, and LINC00657 acts as a competing endogenous RNA and inhibits the expression of miR-590-3p, which then loses its suppression on HIF- $1 \alpha$. The upregulation of HIF- $1 \alpha$ promotes the expression of VEGF, MMP-2, and MMP-9, and eventually induces angiogenesis (Fig. 5). Our present study provides new insight into the roles of LINC00657 and miR$590-3 p$ in oxLDL-induced angiogenesis and may provide a novel strategy for atherosclerosis treatment.

\section{Authorship Contributions}

Participated in research design: Bao, J.-M. Li.

Conducted experiments: Bao, G.-Y. Li, Huang.

Performed data analysis: Tang, Dong.

Wrote or contributed to the writing of the manuscript: Bao, J.M. Li.

\section{References}

Bao MH, Li JM, Luo HQ, Tang L, Lv QL, Li GY, and Zhou HH (2016a) NF- $\kappa$ Bregulated miR-99a modulates endothelial cell inflammation. Mediators Inflamm 2016:5308170

Bao MH, Li JM, Zhou QL, Li GY, Zeng J, Zhao J, and Zhang YW (2016b) Effects of miR-590 on oxLDL-induced endothelial cell apoptosis: roles of p53 and NF- $\kappa$ B. Mol Med Rep 13:867-873.

Björnheden T, Levin M, Evaldsson M, and Wiklund O (1999) Evidence of hypoxic areas within the arterial wall in vivo. Arterioscler Thromb Vasc Biol 19:870-876.

Camaré C, Pucelle M, Nègre-Salvayre A, and Salvayre R (2017) Angiogenesis in the atherosclerotic plaque. Redox Biol 12:18-34.

Chen H, Luo Q, and Li H (2017) MicroRNA-590-3p promotes cell proliferation and invasion by targeting inositol polyphosphate 4-phosphatase type II in human prostate cancer cells. Tumour Biol 39:1-8.

Dandapat A, Hu C, Sun L, and Mehta JL (2007) Small concentrations of oxLDL induce capillary tube formation from endothelial cells via LOX-1-dependent redoxsensitive pathway. Arterioscler Thromb Vasc Biol 27:2435-2442

Deng W, Feng X, Li X, Wang D, and Sun L (2016) Hypoxia-inducible factor 1 in autoimmune diseases. Cell Immunol 303:7-15.

Eulalio A, Mano M, Dal Ferro M, Zentilin L, Sinagra G, Zacchigna S, and Giacca M (2012) Functional screening identifies miRNAs inducing cardiac regeneration. Nature 492:376-381.

Ge X and Gong L (2017) MiR-590-3p suppresses hepatocellular carcinoma growth by targeting TEAD1. Tumour Biol 39:1-8.
Gonzalez-King H, García NA, Ontoria-Oviedo I, Ciria M, Montero JA, and Sepúlveda $\mathrm{P}(2017)$ Hypoxia inducible factor-1 $\alpha$ potentiates Jagged 1-mediated angiogenesis by mesenchymal stem cell-derived exosomes. Stem Cells 35:1747-1759.

Hellings WE, Peeters W, Moll FL, Piers SR, van Setten J, Van der Spek PJ, de Vries JP, Seldenrijk KA, De Bruin PC, Vink A, et al. (2010) Composition of carotid atherosclerotic plaque is associated with cardiovascular outcome: a prognostic study, Circulation 121:1941-1950.

Hutter R, Speidl WS, Valdiviezo C, Sauter B, Corti R, Fuster V, and Badimon JJ (2013) Macrophages transmit potent proangiogenic effects of oxLDL in vitro and in vivo involving HIF- $1 \alpha$ activation: a novel aspect of angiogenesis in atherosclerosis. J Cardiovasc Transl Res 6:558-569.

International Human Genome Sequencing Consortium (2004) Finishing the euchromatic sequence of the human genome. Nature 431:931-945.

Ishigaki Y, Oka Y, and Katagiri H (2009) Circulating oxidized LDL: a biomarker and a pathogenic factor. Curr Opin Lipidol 20:363-369.

Khaidakov M, Mitra S, Wang X, Ding Z, Bora N, Lyzogubov V, Romeo F, Schichman SA and Mehta JL (2012) Large impact of low concentration oxidized LDL on angiogenic potential of human endothelial cells: a microarray study. PLoS One 7:e47421.

Kornienko AE, Guenzl PM, Barlow DP, and Pauler FM (2013) Gene regulation by the act of long non-coding RNA transcription. BMC Biol 11:59.

Lee JW, Bae SH, Jeong JW, Kim SH, and Kim KW (2004) Hypoxia-inducible factor (HIF-1) $\alpha$ : its protein stability and biological functions. Exp Mol Med 36:1-12.

Lee S, Kopp F, Chang TC, Sataluri A, Chen B, Sivakumar S, Yu H, Xie Y, and Mendell JT (2016) Noncoding RNA NORAD regulates genomic stability by sequestering PUMILIO proteins. Cell 164:69-80.

Liu G, Liu M, Wei J, Huang H, Zhang Y, Zhao J, Xiao L, Wu N, Zheng L, and Lin X (2014) CS5931, a novel polypeptide in Ciona savignyi, represses angiogenesis via inhibiting vascular endothelial growth factor (VEGF) and matrix metalloproteinases (MMPs). Mar Drugs 12:1530-1544.

Liu H, Li J, Koirala P, Ding X, Chen B, Wang Y, Wang Z, Wang C, Zhang X, and Mo YY (2016) Long non-coding RNAs as prognostic markers in human breast cancer. Oncotarget 7:20584-20596.

Michalik KM, You X, Manavski Y, Doddaballapur A, Zörnig M, Braun T, John D, Ponomareva Y, Chen W, Uchida S, et al. (2014) Long noncoding RNA MALAT1 regulates endothelial cell function and vessel growth. Circ Res 114:1389-1397.

Parathath S, Mick SL, Feig JE, Joaquin V, Grauer L, Habiel DM, Gassmann M, Gardner LB, and Fisher EA (2011) Hypoxia is present in murine atherosclerotic plaques and has multiple adverse effects on macrophage lipid metabolism. Circ Res 109:1141-1152.

Parma L, Baganha F, Quax PHA, and de Vries MR (2017) Plaque angiogenesis and intraplaque hemorrhage in atherosclerosis. Eur J Pharmacol 816:107-115.

Peluso I, Morabito G, Urban L, Ioannone F, and Serafini M (2012) Oxidative stress in atherosclerosis development: the central role of LDL and oxidative burst. Endocr Metab Immune Disord Drug Targets 12:351-360.

Perrotta I, Moraca FM, Sciangula A, Aquila S, and Mazzulla S (2015) HIF- $1 \alpha$ and VEGF: immunohistochemical profile and possible function in human aortic valve stenosis. Ultrastruct Pathol 39:198-206.

Ribatti D, Nico B, Morbidelli L, Donnini S, Ziche M, Vacca A, Roncali L, and Presta M (2001) Cell-mediated delivery of fibroblast growth factor-2 and vascular endothelial growth factor onto the chick chorioallantoic membrane: endothelial fenestration and angiogenesis. $J$ Vasc Res 38:389-397.

Sun X, Wei L, Chen Q, and Terek RM (2010) CXCR4/SDF1 mediate hypoxia induced chondrosarcoma cell invasion through ERK signaling and increased MMP1 expression. Mol Cancer 9:17.

Tsai KL, Chang YL, Huang PH, Cheng YH, Liu DH, Chen HY, and Kao CL (2016) Ginkgo biloba extract inhibits oxidized low-density lipoprotein (oxLDL)-induced matrix metalloproteinase activation by the modulation of the lectin-like oxLDL receptor 1-regulated signaling pathway in human umbilical vein endothelial cells. J Vasc Surg 63:204-215.e1.

Wang FF, Wang S, Xue WH, and Cheng JL (2016) microRNA-590 suppresses the tumorigenesis and invasiveness of non-small cell lung cancer cells by targeting ADAM9. Mol Cell Biochem 423:29-37.

Yu S, Wong SL, Lau CW, Huang Y, and Yu CM (2011) Oxidized LDL at low concentration promotes in-vitro angiogenesis and activates nitric oxide synthase through PI3K/Akt/eNOS pathway in human coronary artery endothelial cells. Biochem Biophys Res Commun 407:44-48.

Zhou Q, Zhu Y, Wei X, Zhou J, Chang L, Sui H, Han Y, Piao D, Sha R, and Bai Y (2016) MiR-590-5p inhibits colorectal cancer angiogenesis and metastasis by regulating nuclear factor 90/vascular endothelial growth factor A axis. Cell Death Dis 7:e2413.

Address correspondence to: Dr. Mei-Hua Bao, Department of Anatomy, Histology, and Embryology, Science Research Center, Institute of Neuroscience, Changsha Medical University, 1501 Leifeng Road, Wangcheng District, Changsha 410219, China. E-mail: mhbao78@163.com 